\title{
ON THE DISTRIBUTION OF MAXIMA OF MARTINGALES
}

\author{
LESTER E. DUBINS ${ }^{1}$ AND DAVID GILAT ${ }^{2}$
}

\begin{abstract}
Partial order the set of distributions on the real line by $\nu<\nu^{\prime}$ if $\nu(x, \infty)<\nu^{\prime}(x, \infty)$ for all $x$. Then, for each $\mu$ with a finite first moment, the family $M(\mu)$ of all $\nu$ which are distributions of (essential) suprema of martingales closed on the right by a $\mu$-distributed random number, has a least upper bound $\mu^{*}$, and is, therefore, a tight family. In fact, $\mu^{*}$ is $\bar{\mu}$, the distribution of the Hardy-Littlewood extremal maximal function associated with $\mu$. Moreover, $\mu^{*}$ is itself an element of $M(\mu)$. For each $p>1$, the classical moment inequality that the $L_{p}$ norm of $\bar{\mu}$ (and of $\mu^{*}$ ) is at most $p /(p-1)$ times the $L_{p}$ norm of $\mu$ is shown to be sharp.
\end{abstract}

Let $f$ be the essentially unique, nondecreasing, function on the unit interval whose distribution with respect to Lebesgue measure is the given distribution, $\mu$. Since $\mu$ is assumed to have a finite first moment, $\int_{0}^{1}|f|$, its extremal, Hardy-Littlewood [5] maximal function, $H$, is well defined by

$$
H(t)=\frac{1}{1-t} \int_{t}^{1} f(s) d s, \quad 0 \leqslant t<1 .
$$

Of course, $\bar{\mu}(x, \infty)$ is the length of the subinterval of $(0,1)$ consisting of all $t$ such that $H(t)>x$.

LEMMA 1. $\bar{\mu}$ is an upper bound for $M(\mu)$.

Proof. For $\mu$ supported by the nonnegative reals, Lemma 1 is merely a reformulation of [1, Theorem 3a]. Moreover, the proof given there applies almost without change if the assumption of nonnegativity is dropped.

LEMMA 2. $\bar{\mu} \in M(\mu)$.

Proof. For each $s$ in the open unit interval $(0,1)$, consider that function on the closed unit interval which agrees with $H$ to the left of $s$ and which equals $f(s)$ to the right of $s$. That is, with the convenient notation introduced by de Finetti, [2, p. xviii] and [3, Chapter 2, \$2.11] define:

$$
Y_{t}(s)=H(t)(t \leqslant s)+f(s)(t>s), \quad 0 \leqslant t \leqslant 1 .
$$

As is not difficult to verify:

(a) $Y_{1}=f$;

Received by the editors August 8, 1977.

AMS (MOS) subject classifications (1970). Primary 60G45.

Key words and phrases. Hardy-Littlewood maximal function, martingale, $L_{p}, L \log L$.

'This research was sponsored by National Science Foundation Grant No. MCS75-09459-A01.

${ }^{2}$ Work was done at the University of California, Berkeley and University of Minnesota, while on leave. 
(b) $\sup Y_{t}(s)(0 \leqslant t \leqslant 1)=H(s) ; 0<s<1$;

(c) $Y$ is a martingale, where, of course, the open unit interval endowed with Lebesgue measure is the underlying probability space. To verify (c), it is necessary to show that $E\left(Y_{1} \mid Y_{s}, s \leqslant t\right)$ equals $Y_{t}$, which is most simply done by checking separately that each is equal to $E\left(Y_{1} \mid Y_{t}\right)$. The function $H$ is not only the sup of the $Y_{t}$, as is asserted by (b); it is also (a version of) the essential sup of the $Y_{t}$. So $Y$ has an essential sup which is $\bar{\mu}$-distributed. Hence $\bar{\mu} \in M(\mu)$.

Trivially, $M(\mu)$ is bounded below, say by $\mu$, which, incidentally, obviously belongs to $M(\mu)$. Since a set of distributions on the real line is tight if, and only if, it is bounded above and below, $M(\mu)$ is tight.

In summary:

THEOREM 1. For each $\mu$ with a finite mean, $M(\mu)$ is tight and has the least upper bound, $\mu^{*}$, namely the Hardy-Littlewood distribution $\bar{\mu}$ associated to $\mu$. Moreover, $\bar{\mu}$ is an element of $M(\mu)$, as is the greatest lower bound of $M(\mu)$, namely $\mu$ itself.

Of course, since $\mu^{*}=\bar{\mu}$, all inequalities known to hold for $\bar{\mu}$ automatically hold for $\mu^{*}$. In particular, Hardy and Littlewood [5] have shown that for $p>1$, the $L_{p}$ norm of $\bar{\mu}$ is at most $(p /(p-1))$ times the $L_{p}$ norm of $\mu$. This provides an alternative proof of Doob's $[4$, p. 317] inequalities to the effect that the $L_{p}$ norm of $\mu^{*}$ is at most $(p /(p-1))$ times the $L_{p}$ norm of $\mu$.

THEOREM 2. The above moment inequalities of Hardy-Littlewood, and of Doob, are sharp.

Proof. For $c>1$, let $f(t)=1 /(1-t)^{1 / c}, 0 \leqslant t<1$. Then $f$ is in every $L_{p}$ with $1<p<c$, and an elementary computation reveals that $H=(c /(c-$ 1)) $f$. Consequently,

$$
\int H^{p}=\left(\frac{c}{c-1}\right)^{p} \int f^{p} .
$$

If, in (3), $c$ converges downward to $p$, the asserted sharpness is obtained.

Presumably the inequalities, though sharp, need not be attained, but we do not pursue this matter.

The problem of sharpness for the corresponding inequalities when $\mu$ is in $L \log L$ rather than $L_{p}$ has not yielded its mysteries to us.

\section{REFERENCES}

1. D. Blackwell and L. E. Dubins, A converse to the dominated convergence theorem, Illinois J. Math. 7 (1963), 508-514.

2. B. de Finetti, Probability, induction and statistics, Wiley, New York, 1972.

3. Theory of probability, Vol. 1, Wiley, New York, 1974.

4. J. L. Doob, Stochastic processes, Wiley, New York, 1953.

5. G. H. Hardy and J. E. Littlewood, A maximal theorem with function-theoretic applications, Acta Math. 54 (1930), 81-116.

Department of Mathematics, University of California, Berkeley, California 94720

Department of Mathematics, Tel Aviv University, Tel Aviv, Israel 